\title{
Quandles and Linking Number
}

\author{
Natasha Harrell \\ natasha@math.ucr.edu
}

\author{
Sam Nelson \\ knots@esotericka.org
}

Department of Mathematics, University of California, Riverside

900 University Avenue, Riverside, CA, 92521

\begin{abstract}
We study the quandle counting invariant for a certain family of finite quandles with trivial orbit subquandles. We show how these invariants determine the linking number of classical two-component links up to sign.
\end{abstract}

KEYWORDS: Virtual links, linking number, finite quandles

2000 MSC: 57M27, 55M25

\section{Introduction}

In the study of finite quandles and the knot invariants they define, decomposable quandles are often neglected in favor of indecomposable or connected quandles since for knots, two decomposable quandles with the same orbit decomposition define the same counting invariant: if $Q=Q_{1} \cup Q_{2}$ then

$$
\#(\operatorname{Hom}(Q(K), Q))=\#\left(\operatorname{Hom}\left(Q(K), Q_{1}\right)\right)+\#\left(\operatorname{Hom}\left(Q(K), Q_{2}\right)\right),
$$

the sum of the counting invariants defined by the orbit subquandles, where $\#(X)$ denotes the cardinality of the set $X$.

This follows from the fact that knot quandles have a single orbit, so the image of any homomorphism $\phi: Q(K) \rightarrow T$ from a knot quandle $K$ into a finite target quandle $T$ must lie inside a single orbit. The quandle of a multi-component link, however, is not connected, and the counting invariants defined by nonisomorphic decomposable quandles with the same orbit decomposition are generally different for links.

The motivation for this paper was to study the counting invariants defined by decomposable quandles for multi-component virtual links. We started with the case of finite target quandles whose orbits are trivial subquandles, so that all of the interesting structure is in the off-diagonal portion of the quandle's matrix - trivial subquandles which have been put together in a non-trivial way. We call such quandles trivial orbit quandles or TOQs.

Our main result says that the linking number of a two-component classical link is determined up to sign by the counting invariants for a family of trivial orbit quandles of a particular form.

The paper is organized as follows: in section 2 we review the definitions of quandles, the quandle counting invariant and virtual linking numbers. In section 3 we give our main result and some examples. 


\section{Quandles and the counting invariant}

In this section we review some definitions.

Definition 1 A virtual link diagram is a (possibly non-planar) 4-valent graph with vertices decorated with crossing information, i.e., two edges meet at a vertex to form an over-strand and the other two form an under-strand. A virtual link is an equivalence class of virtual link diagrams under the equivalence relation generated by the Reidemeister moves (I, II and III). An oriented virtual link diagram has a preferred direction of travel around each component indicated by arrows. To draw non-planar virtual links on planar paper, we introduce virtual crossings $(\$)$ to distinguish the crossings arising from non-planarity from the ordinary classical crossings. Two virtual link diagrams are equivalent or virtually isotopic iff they are related by a finite sequence of virtual Reidemeister moves:

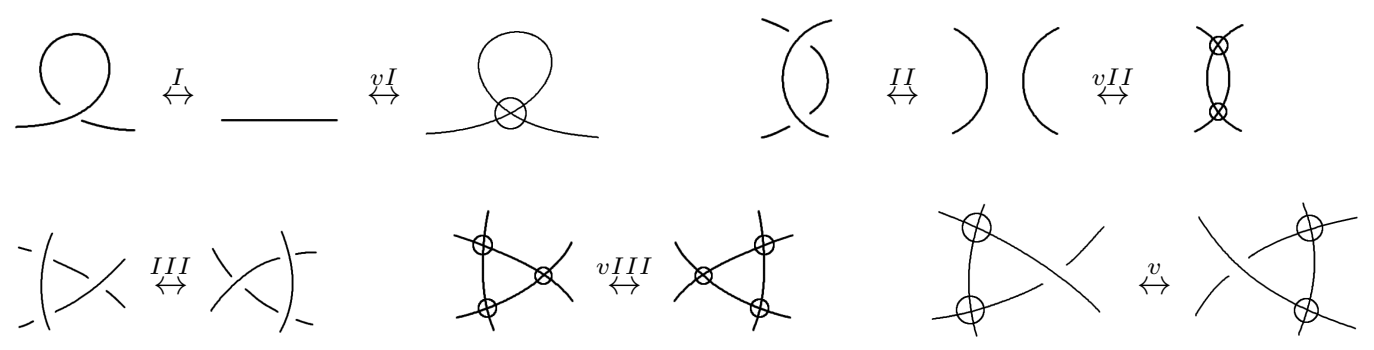

Classical knot theory may be regarded as a special case of virtual knot theory, since virtually isotopic classical knots are ambient isotopic (see [6], 4], and [7]). In particular, invariants of and theorems about virtual knots are invariants of and theorems about classical knots.

Next, we need

Definition 2 A quandle is a set $Q$ endowed with a binary operation $\triangleright: Q \times Q \rightarrow Q$ satisfying

(i) For all $a \in Q, a \triangleright a=a$,

(ii) For all $a, b \in Q$, there exists a unique $c \in Q$ such that $a=c \triangleright b$, and

(iii) For all $a, b, c \in Q,(a \triangleright b) \triangleright c=(a \triangleright c) \triangleright(b \triangleright c)$.

The uniqueness in axiom (ii) gives us an inverse quandle operation $\triangleright^{-1}$ (also denoted by $\bar{\triangleright}$ or $\triangleleft$ ), called the dual operation.

As expected, a homomorphism of quandles is a map $f: Q \rightarrow Q^{\prime}$ such that

$$
f(a \triangleright b)=f(a) \triangleright f(b) \quad \forall a, b \in Q .
$$

We may specify a quandle structure on the finite set $Q=\left\{x_{1}, x_{2}, \ldots, x_{n}\right\}$ by giving an $n \times n$ matrix $M_{Q}$ whose entry in row $i$ column $j$ is $k$ where $x_{k}=x_{i} \triangleright x_{j}$; that is, $M_{Q}$ is the operation table of $(Q, \triangleright)$ where we forget the " $x$ "s and just write the subscripts. 
Example 1 The trivial quandle of order $n$, denoted $T_{n}$, is the quandle with matrix

$$
M=\left[\begin{array}{cccc}
1 & 1 & \ldots & 1 \\
2 & 2 & \ldots & 2 \\
\vdots & \vdots & & \vdots \\
n & n & \ldots & n
\end{array}\right]
$$

Quandles and their structure have been studied in many works such as [5, 2], 1] and more. A quandle $Q$ is decomposable if we may write $Q=Q_{1} \cup Q_{2}$ where $Q_{1} \cap Q_{2}=\emptyset$ and $Q_{1}, Q_{2}$ are both subquandles of $Q$. Note that even if a quandle is not decomposable in this sense, we may still be able to write $Q=\bigcup_{i=1}^{n} Q_{i}$ for $Q_{i}$ disjoint subquandles; see [1] and [8. A quandle is connected if there is an element $a \in Q$ such that every element of $Q$ is equivalent to a word of the form

$$
\left(\ldots\left(\left(a \triangleright^{ \pm 1} x_{1}\right) \triangleright^{ \pm 1} x_{2}\right) \triangleright^{ \pm 1} \ldots\right) \triangleright^{ \pm 1} x_{n} .
$$

In [5] (see also 2]), a quandle is defined for any oriented tame classical link $L$ via a Wirtingertype presentation, which is called the knot quandle of the link $L$. Specifically, looking in the positive direction of the overcrossing strand $b$, if the undercrossing arc on the right is $a$ then the one on the left is $a \triangleright b$. Note that we do not use the orientation of the undercrossing strand. a

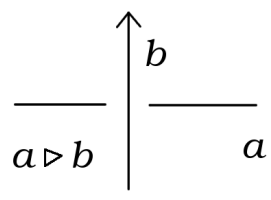

Figure 1: Quandle generators at a crossing

The isomorphism type of the knot quandle is invariant under Reidemeister moves; indeed, the quandle axioms are chosen specifically to make this so. If $L$ is a knot and $x$ is a generator assigned to an $\operatorname{arc}$ in $L$, then every generator (and hence every word) in the knot quandle is equivalent to a word starting with $x$ since we can just proceed around the knot following the specified orientation, picking up quandle operations to build a word, until we reach $x$. Hence, the knot quandle of a single-component link is connected.

While Joyce defined the knot quandle only for classical knots and links, in [6] Kauffman observed that the same Wirtinger-type definition extends to virtual knots and links by simply ignoring virtual crossings. The resulting quandle, while not a classifying invariant for virtual knots and links, is nevertheless an invariant of virtual isotopy.

Given a finite quandle $T$, the number of homomorphisms from a knot quandle $Q(L)$ into $T$ is an easily computable link invariant: since $Q(L)$ is finitely generated, there is a finite set of possible homomorphisms into $T$, so we can simply check which of these satisfy the relations in $Q(L)$ and hence are homomorphisms. The following is a standard result:

Theorem 1 Let $L$ be a link with knot quandle $Q(L)$ and let $T$ be a finite quandle. Then the number of homomorphisms \# $\operatorname{Hom}(Q(L), T))$ is an invariant of quandle isomorphism and hence is an invariant of virtual isotopy of the link. 
Indeed, Joyce [5] showed that the knot quandle determines both the knot group and a longitude, and hence determines the knot type of a classical knot up to homeomorphism (not necessarily orientation preserving) of pairs $\left(S^{3}, L\right)$. Hence, many of the classical link invariants should be derivable from the knot quandle. A deeper understanding of how link invariants arise from the knot quandle should provide us with a better understanding both of how other link invariants are related and of links themselves. In the next section we will show how linking number is determined up to sign by the quandle counting invariants associated to a particular kind of non-connected quandle.

\section{Quandle counting and linking number}

We begin with a discussion of linking number for virtual knots.

In 4 ] we find the following definition:

Definition 3 Let $L=L_{1} \cup L_{2}$ be an oriented virtual link diagram with two components $L_{1}$ and $L_{2}$. Then $l k_{1 / 2}\left(L_{1}, L_{2}\right)$ is the sum of crossing signs at crossings where $L_{1}$ crosses over $L_{2}$, and $l k_{2 / 1}\left(L_{1}, L_{2}\right)$ is the sum of crossing signs at crossings where $L_{2}$ crosses over $L_{1}$. These are the virtual linking numbers of $L_{1}$ with $L_{2}$. The usual (classical) linking number $l k\left(L_{1}, L_{2}\right)$ satisfies

$$
l k\left(L_{1}, L_{2}\right)=\frac{l k_{1 / 2}\left(L_{1}, L_{2}\right)+l k_{2 / 1}\left(L_{1}, L_{2}\right)}{2} .
$$

These virtual linking numbers are invariants of virtual isotopy, since the only virtual move which might potentially change them, move II, is seen to preserve them by inspection.

For classical links, the two linking numbers are equal since if $L_{1}$ crosses over $L_{2}$ at a positive crossing, it must either cross back over $L_{2}$ at a negative crossing (contributing zero to both linking numbers) or cross under $L_{2}$ at a positive crossing (contributing +1 to both linking numbers) to come back to join itself without any virtual crossings. For virtual links, the two linking numbers are completely independent. Indeed, we have

Proposition 2 Let $L=L_{1} \cup L_{2}$ be a virtual link. If

$$
l k_{1 / 2}\left(L_{1}, L_{2}\right) \neq l k_{2 / 1}\left(L_{1}, L_{2}\right)
$$

then $L$ is non-classical.

For virtual links, the virtual linking numbers have some advantages over the usual classical linking number. For example, if $L=L_{1} \cup L_{2}$ is split, then both $l k_{1 / 2}\left(L_{1}, L_{2}\right)=0$ and $l k_{2 / 1}\left(L_{1}, L_{2}\right)=0$; this is a stronger condition than $l k\left(L_{1}, L_{2}\right)=0$, since contributions from two unequal virtual linking numbers may cancel.

Example 2 The virtual linking numbers detect the fact that virtual link

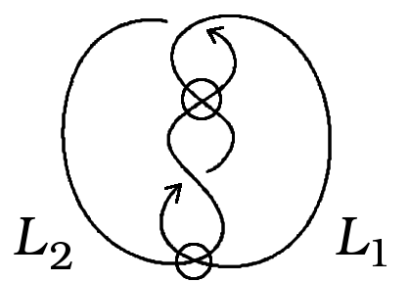


is non-split since $l k_{1 / 2}\left(L_{1}, L_{2}\right)=+1$ while $l k_{2 / 1}\left(L_{1}, L_{2}\right)=-1$. The fact that $l k_{1 / 2}\left(L_{1}, L_{2}\right) \neq$ $l k_{2 / 1}\left(L_{1}, L_{2}\right)$ says that $L$ is non-classical. However, the classical linking number $l k\left(L_{1}, L_{2}\right)=0$.

Definition 4 Let $X_{n}$ be the set $\{1,2, \ldots, n+1\}$ with operation $i \triangleright j=M_{i j}$ defined by the matrix

$$
M=\left[\begin{array}{ccccc}
1 & 1 & \ldots & 1 & 2 \\
2 & 2 & \ldots & 2 & 3 \\
\vdots & \vdots & & \vdots & \vdots \\
n-1 & n-1 & \ldots & n-1 & n \\
n & n & \ldots & n & 1 \\
n+1 & n+1 & \ldots & n+1 & n+1
\end{array}\right] .
$$

Proposition $3 X_{n}$ is a quandle.

Remark 1 Note that $X_{n}$ has two orbit subquandles $S_{n} \cong T_{n}$ and $S_{n+1} \cong T_{1}$, both of which are trivial quandles, and we have

$$
a \triangleright b= \begin{cases}a+1 \bmod n & a \in S_{n}, b=n+1 \\ a & \text { otherwise }\end{cases}
$$

and

$$
a \triangleright^{-1} b= \begin{cases}a-1 \bmod n & a \in S_{n}, b=n+1 \\ a & \text { otherwise. }\end{cases}
$$

\section{Proof.}

As noted in 3 , the first two quandle axioms are easy to check since the diagonal entries are $1,2,3, \ldots, n+1$ in order and each column is a permutation of $\{1,2, \ldots, n+1\}$. Thus, we need only check that the third quandle axiom is satisfied. We do this in cases.

Case 1: $a, b, c$ all in the same subquandle. In this case,

$$
(a \triangleright b) \triangleright c=(a \triangleright c) \triangleright(b \triangleright c)
$$

since the subquandles are quandles themselves.

Case 2: $a, b$ in one orbit, $c$ in the other. There are two subcases.

(i) If $a, b \in S_{n}$ and $c \in S_{n+1}$ then we have

$$
(a \triangleright b) \triangleright c=a \triangleright c=a+1 \bmod n
$$

and

$$
(a \triangleright c) \triangleright(b \triangleright c)=(a+1 \bmod n) \triangleright(b+1 \bmod n)=a+1 \bmod n .
$$

(ii) If $a, b \in S_{n+1}$ and $c \in S_{n}$, then

$$
(a \triangleright b) \triangleright c=a \triangleright c=a
$$

and

$$
(a \triangleright c) \triangleright(b \triangleright c)=a \triangleright b=a .
$$

Case 3: $b, c$ in one orbit and $a$ in the other. Again we check both subcases. 
(i) If $a \in S_{n}$ and $b, c \in S_{n+1}$ then

$$
(a \triangleright b) \triangleright c=(a+1 \bmod n) \triangleright c=a+2 \bmod n
$$

and

$$
(a \triangleright c) \triangleright(b \triangleright c)=(a+1 \bmod n) \triangleright c=a+2 \bmod n .
$$

(ii) If $b, c \in S_{n}$ and $a \in S_{n+1}$ then

$$
(a \triangleright b) \triangleright c=a \triangleright c=a
$$

and

$$
(a \triangleright c) \triangleright(b \triangleright c)=a \triangleright b=a .
$$

Case 4: $a, c$ in one orbit and $b$ in the other.

(i) If $a, c \in S_{n}$ and $b=S_{n+1}$ then

$$
(a \triangleright b) \triangleright c=(a+1 \bmod n) \triangleright c=a+1 \bmod n
$$

and

$$
(a \triangleright c) \triangleright(b \triangleright c)=a \triangleright b=a+1 \bmod n .
$$

(ii) If $a, c \in S_{n+1}$ and $b \in S_{n}$ then

$$
(a \triangleright b) \triangleright c=a \triangleright c=a
$$

and

$$
(a \triangleright c) \triangleright(b \triangleright c)=a \triangleright(b+1 \bmod n)=a .
$$

Definition 5 A coloring of $L$ by $T$ is a homomorphism of quandles $C: Q(L) \rightarrow T$ regarded as an assignment of a "color" $x \in T$ to each arc in $L$ such that the quandle condition pictured in figure 1 is satisfied at every crossing.

Example 3 Every link $L=L_{1} \cup \cdots \cup L_{m}$ has $n^{m}$ colorings by $T_{n}$, the trivial quandle of order $\mathrm{n}$. Since $a \triangleright b=a$ for every $b \in T_{n}$, all arcs in the same component must have the same color in any coloring, and we can choose the colors for each component arbitrarily since every color acts trivially on every other color.

This example implies that the number of colorings of a link $L$ by $X_{n}$ is at least

$$
\#\left(\operatorname{Hom}\left(Q(L), S_{n}\right)\right)+\#\left(\operatorname{Hom}\left(Q(L), S_{n+1}\right)\right)=n^{2}+1 .
$$

We will now investigate the actual number of colorings of a virtual link $L$ by $X_{n}$.

For the remainder of this paper, let $L$ be a two-component virtual link diagram $L=L_{1} \cup L_{2}$. To find quandle colorings of $L$, we take a choice of color $x \in X_{n}$ for one arc and attempt to "push it through" each crossing, that is, assign to the opposite undercrossing arc the color determined by the quandle coloring condition and the color on the overcrossing arc. Since our coloring quandle $X_{n}$ is a trivial orbit quandle, pushing any color through at a crossing where both strands are from the same component yields the same color on both underarcs since the colors on a component are from the same trivial orbit subquandle. Thus we have: 
Lemma 4 In a quandle coloring of a link by $X_{n}$ (or any trivial orbit quandle), the colors on the undercrossing strands at a crossing can differ only if the overcrossing and undercrossing strands belong to distinct components.

The quandle operations in $X_{n}$ imply the following observation.

Lemma 5 In a quandle coloring of $L$ by $X_{n}$, an inbound undercrossing arc colored $x \in S_{n}$ pushes through a crossing with an overarc colored $n+1$ to become $x+1 \bmod n$ at a positive crossing and $x-1 \bmod n$ at a negative crossing.

We can get an easy upper bound for \#(Hom $\left.\left(Q(L), X_{n}\right)\right)$ :

Proposition 6 L has at most $(n+1)^{2}$ colorings by $X_{n}$.

Proof. Pushing through any choice of color for a starting arc extends the same color along that component of the link until overcrossings from the other component of $L$ are reached. For any pair of starting arcs with one arc on each component, if both starting colors are from the same orbit, then both colors push through unchanged and we have a coloring of the link which is constant on each component. If the colors are from different orbits in $X_{n}$, then one component has every arc colored $n+1$, and a given starting coloring $a \in S_{n}$ pushes through an overcrossing colored with $n+1$ to become $a+1 \bmod n$ or $a-1 \bmod n$ depending on the crossing sign. Continuing pushing through each crossing, we eventually reach our original starting arc. Then we have a valid quandle coloring iff the color thus determined on the starting arc agrees with the original color. In both cases (starting arcs in the same orbit subquandle or in different orbit subquandles), the colors on all of the arcs are determined by the colors on the initial two arcs. There are $(n+1)^{2}$ such choices.

In particular, we have

Lemma $7 L$ has a coloring by $X_{n}$ with $n+1$ on $L_{i}$ and colors from $S_{n}$ on $L_{j}$ iff

$$
l k_{i / j}\left(L_{i}, L_{j}\right) \equiv 0 \bmod n .
$$

Moreover, if $L$ has one such coloring, then it has $n$ such colorings.

Proof. If we color one arc of $L_{i}$ with $n+1 \in X_{n}$, then in any quandle coloring of $L$ every arc of $L_{i}$ must be colored with $n+1$, since every element of $X_{n}$ acts trivially on $n+1$. In order to have a valid quandle coloring, the original color on the starting arc must match the color determined by pushing $x$ through the full length of the component to return to the starting arc. Lemma 5 implies that this final color is $x+l k_{i / j}\left(L_{i}, L_{j}\right) \bmod n$, so an initial color of $x \neq n+1$ on an arc of $L_{j}$ pushes through to a valid coloring iff $l k_{i / j}\left(L_{i}, L_{j}\right) \equiv 0 \bmod n$. Finally, note that the property of pushing through does not depend on the actual value of $x \neq n+1$, only its orbit, so if one such color yields a valid quandle coloring, then all $n$ colors in $S_{n}$ yield valid quandle colorings.

Corollary 8 If $L=L_{1} \cup L_{2}$ then

$$
\#\left(\operatorname{Hom}\left(Q(L), X_{n}\right)\right) \in\left\{(n+1)^{2},(n+1)^{2}-n, n^{2}+1\right\} .
$$


Proof. We have \# $\left(\operatorname{Hom}\left(Q(L), X_{n}\right)\right)=(n+1)^{2}$ if $L$ has colorings with $n+1$ on $L_{i}$ and colors from $S_{n}$ on $L_{j}$ for both $i=1,2 ; \#\left(\operatorname{Hom}\left(Q(L), X_{n}\right)\right)=(n+1)^{2}-n=n^{2}+n+1$ if $L$ has colorings with $n+1$ on $L_{i}$ and colors in $S_{n}$ on $L_{j}$ for $i=1$ or $i=2$ but not both; and \# $\left.\operatorname{Hom}\left(Q(L), X_{n}\right)\right)=n^{2}+1$ if $L$ has no multi-orbit colorings. Note that $\#\left(\operatorname{Hom}\left(Q(L), X_{n}\right)\right)=(n+1)^{2}-n$ implies that the virtual linking numbers are unequal and $L$ is non-classical.

Corollary $9 l k_{1 / 2}\left(L_{1}, L_{2}\right)=l k_{2 / 1}\left(L_{1}, L_{2}\right)=0$ iff

$$
\#\left(\operatorname{Hom}\left(Q(L), X_{n}\right)\right)=(n+1)^{2} \text { for all } n \geq 2 .
$$

Corollary 10 Let $n \in \mathbb{Z}$ with $n \geq 2$. If

$$
\left|l k_{1 / 2}\left(L_{1}, L_{2}\right)\right|+\left|l k_{2 / 1}\left(L_{1}, L_{2}\right)\right| \neq 0
$$

then

$$
\#\left(\operatorname{Hom}\left(Q(L), X_{n}\right)\right)=(n+1)^{2} \Longleftrightarrow n|| l k_{1 / 2}\left(L_{1}, L_{2}\right) \mid \text { and } n|| l k_{2 / 1}\left(L_{1}, L_{2}\right) \mid \text {. }
$$

Proof. \#(Hom $\left.\left(Q(L), X_{n}\right)\right)=(n+1)^{2}$ if and only if each starting coloring choice for a given pair of arcs on the two components pushes through to a valid coloring. By lemma 7 , this can only happen if both virtual linking numbers are zero $\bmod n$, i. e., if $n|| l k_{1 / 2}\left(L_{1}, L_{2}\right) \mid$ and $n|| l k_{2 / 1}\left(L_{1}, L_{2}\right) \mid$.

\section{Corollary 11 If}

$$
\left|l k_{1 / 2}\left(L_{1}, L_{2}\right)\right|+\left|l k_{2 / 1}\left(L_{1}, L_{2}\right)\right| \neq 0
$$

then $\#\left(\operatorname{Hom}\left(Q(L), X_{n}\right)\right)<(n+1)^{2}$ for any $n$ greater than the number of two-component crossings in the link diagram $L$.

Corollary 12 If either $\left|l k_{1 / 2}\left(L_{1}, L_{2}\right)\right|=1$ or $\left|l k_{2 / 1}\left(L_{1}, L_{2}\right)\right|=1$ then $\#\left(\operatorname{Hom}\left(Q(L), X_{n}\right)\right)<(n+1)^{2}$ for all $n \geq 2$. Conversely, if $\#\left(\operatorname{Hom}\left(Q(L), X_{n}\right)\right)<(n+1)^{2}$ for all $n \geq 2$ then either $\left|l k_{1 / 2}\left(L_{1}, L_{2}\right)\right|=$ $1,\left|l k_{2 / 1}\left(L_{1}, L_{2}\right)\right|=1$ or both $\left|l k_{1 / 2}\left(L_{1}, L_{2}\right)\right|=\left|l k_{2 / 1}\left(L_{1}, L_{2}\right)\right|=1$.

Proof. If $\left|l k_{i / j}\left(L_{1}, L_{2}\right)\right|=1$ then $\left|l k_{1 / 2}\left(L_{1}, L_{2}\right)\right|+\left|l k_{1 / 2}\left(L_{1}, L_{2}\right)\right| \neq 0$. Then $n \geq 2$ in corollary 10 implies that \# $\left(\operatorname{Hom}\left(Q(L), X_{n}\right)\right)<(n+1)^{2}$, since $n \nmid 1$.

Conversely, suppose \#(Hom $\left.\left(Q(L), X_{n}\right)\right)<(n+1)^{2}$ for all $n \geq 2$. Then by corollary 9 at least one of the two virtual linking numbers is greater than 0 in absolute value. If both are greater than 1 in absolute value, then let $N=\operatorname{gcd}\left(\left|l k_{1 / 2}\left(L_{1}, L_{2}\right)\right|,\left|l k_{2 / 1}\left(L_{1}, L_{2}\right)\right|\right)$. Then by lemma 7 if $N>1$ then $\#\left(\operatorname{Hom}\left(Q(L), X_{N}\right)\right)=(N+1)^{2}$, contrary to hypothesis. Hence either $\left|l k_{1 / 2}\left(L_{1}, L_{2}\right)\right|=1$, $\left|l k_{2 / 1}\left(L_{1}, L_{2}\right)\right|=1$, or both $\left|l k_{1 / 2}\left(L_{1}, L_{2}\right)\right|=\left|l k_{2 / 1}\left(L_{1}, L_{2}\right)\right|=1$.

Theorem 13 Let $L=L_{1} \cup L_{2}$ be a virtual link diagram and let

$$
\begin{aligned}
S & =\left\{n: \#\left(\operatorname{Hom}\left(Q(L), X_{n}\right)\right)=(n+1)^{2}, n \geq 2\right\} \text { and } \\
S^{\prime} & =\left\{n: \#\left(\operatorname{Hom}\left(Q(L), X_{n}\right)\right)=(n+1)^{2}-n, n \geq 2\right\} .
\end{aligned}
$$

Then if $0<\#(S)<\infty$ we have

$$
\max (S)=\operatorname{gcd}\left(\left|l k_{1 / 2}\left(L_{1}, L_{2}\right)\right|,\left|l k_{2 / 1}\left(L_{1}, L_{2}\right)\right|\right)
$$

and

$$
\max \left(S^{\prime}\right)=\max \left(\left\{n: n|| l k_{i / j}\left(L_{1}, L_{2}\right) \mid \text { and } n \backslash\left|l k_{j / i}\left(L_{1}, L_{2}\right)\right|\right\}\right)
$$


Proof. If $0<\#(S)<\infty$ then neither linking number is 0 or 1 in absolute value by corollaries 9 and 12. Then $n \in S^{\prime}$ says that $n$ divides one linking number but not the other by lemma $7 \max (S)$ is the largest number which divides both $\left|l k_{1 / 2}\left(L_{1}, L_{2}\right)\right|$ and $\left|l k_{2 / 1}\left(L_{1}, L_{2}\right)\right|$ by corollary 10 .

We can now state our main result, which says that the classical linking number is determined up to sign by the quandle coloring invariants with target quandle $X_{n}$ for $n \geq 2$.

Theorem 14 Let $L=L_{1} \cup L_{2}$ be a classical link diagram and let

$$
S=\left\{n: \#\left(\operatorname{Hom}\left(Q(L), X_{n}\right)\right)=(n+1)^{2}, n \geq 2\right\} .
$$

Then

$$
\left|l k\left(L_{1}, L_{2}\right)\right|=\frac{\left|l k_{1 / 2}\left(L_{1}, L_{2}\right)\right|+\left|l k_{2 / 1}\left(L_{1}, L_{2}\right)\right|}{2}= \begin{cases}0, & \#(S)=\infty, \\ 1, & \#(S)=0, \text { or } \\ \max (S), & 0<\#(S)<\infty\end{cases}
$$

Proof. If both virtual linking numbers are zero, then corollary 9 says $S=\{n \in \mathbb{Z} \mid n \geq 2\}$. If both are \pm 1 , then by corollary 12 we have $S=\emptyset$. Finally, if $\left|l k_{1 / 2}\left(L_{1}, L_{2}\right)\right|=m$ (which equals $\left|l k_{2 / 1}\left(L_{1}, L_{2}\right)\right|$ since $L$ is classical) then the set $S$ includes all and only positive integer divisors of $m$, so $m=\max (S)$.

\section{Example 4}

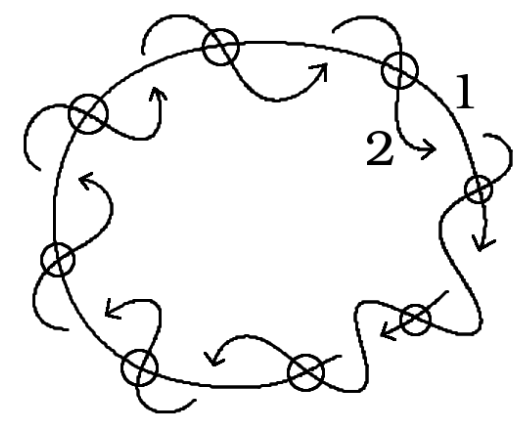

\begin{tabular}{|c|c|}
\hline$n$ & $\operatorname{Hom}\left(Q(L), X_{n}\right)$ \\
\hline 2 & $9=(n+1)^{2}$ \\
3 & $13=(n+1)^{2}-n$ \\
4 & $17=(n+1)^{2}-2 n$ \\
5 & $26=(n+1)^{2}-2 n$ \\
6 & $43=(n+1)^{2}-n$ \\
7 & $50=(n+1)^{2}-2 n$ \\
\hline
\end{tabular}

This virtual link has two components labeled 1 and 2 , with virtual linking numbers $l k_{1 / 2}(1,2)=6$ and $l k_{2,1}(1,2)=-2$. Then $\operatorname{gcd}\left(\left|l k_{1 / 2}\left(L_{1}, L_{2}\right)\right|,\left|l k_{2 / 1}\left(L_{1}, L_{2}\right)\right|\right)=2$ and the largest cardinality which divides one linking number but not both is 6 . Our Maple computations confirm the values in the table.

As a practical observation, we note that corollary 11 implies that it is not necessary to test an infinite number of quandle counting invariants to determine \# $(S)$; it is sufficient to compute $\#\left(\operatorname{Hom}\left(Q(L), X_{n}\right)\right)$ for $2 \leq n \leq N$ where $N$ is the number of multi-component crossings in $L$. Of course, our interest in theorem 14 lies not in finding a new less practical method of computing linking numbers but rather as a connection between quandle-based link invariants and classical link invariants. 


\section{References}

[1] N. Andruskiewitsch and M. Graña. From racks to pointed Hopf algebras. Adv. Math. 178 (2003) $177-243$.

[2] R. Fenn and C. Rourke. Racks and links in codimension two. J. Knot Theory Ramifications 1 (1992) 343-406.

[3] B. Ho and S. Nelson. Matrices and finite quandles. Homol. Homotop. Appl. 7 (2005) 197-208.

[4] M. Goussarov, M. Polyak and O. Viro. Finite-type invariants of classical and virtual knots. Topology 39 (2000) 1045-1068.

[5] D. Joyce. A classifying invariant of knots, the knot quandle. J. Pure Appl. Algebra 23 (1982) $37-65$.

[6] L. H. Kauffman. Virtual Knot Theory. European J. Combin. 20 (1999) 663-690.

[7] G. Kuperberg. What is a virtual link? Algebr. Geom. Topol. 3 (2003) 587-591.

[8] S. Nelson and C-Y. Wong. On the orbit decomposition of finite quandles. J. Knot Theory Ramifications 15 (2006) 761-772. 